\title{
Platelet-Derived Growth Factor-BB Mediates Cell Migration through Induction of Activating Transcription Factor 4 and Tenascin-C
}

\author{
Kristine P. Malabanan, Anjali V. Sheahan, and \\ Levon M. Khachigian \\ From the Centre for Vascular Research, University of New South \\ Wales, Sydney, Australia
}

The acute response to vascular cell injury, which underpins vasculo-occlusive pathologies such as atherogenesis and restenosis after percutaneous coronary intervention, involves a complex series of molecular events that alter patterns of gene expression and favor a synthetic phenotype. One transcription factor that has been implicated in this process is the evolutionarily conserved mammalian stress response pathway regulator activating transcription factor 4 (ATF4). Here, we show for the first time that both mRNA and protein levels of ATF- 4 are induced in smooth muscle cells (SMCs) by the potent migratory factor PDGF-BB through PDGFR- $\beta$. PDGF-BB also stimulates the expression of tenascin-C (TN-C), an extracellular matrix glycoprotein that regulates the activity of focal adhesion complexes, facilitating the SMC migration that underlies negative vascular remodeling in response to injury. Overexpression of ATF- 4 increased transcript levels of the four TN-C isoforms in rat vascular SMCs, and ATF-4 knockdown inhibited PDGFBB-inducible TN-C expression in vitro and injury-inducible TN-C protein expression in the balloon-injured rat artery wall. Furthermore, we show that ATF-4 is required for PDGF-BB-inducible SMC migration in response to injury. PDGF-BB-induced migration was also compromised in ATF-4 null mEFs, and this effect was rescued by the addition of TN-C. Our findings thus demonstrate the role of ATF- 4 in both injury- and PDGF-BB-inducible TN-C expression and cell migration. (Am J Patbol 2012, 180:2590-2597; bttp:// dx.doi.org/10.1016/j.ajpath.2012.02.009)

The response to vascular cell injury is a complex process of transcription and vascular remodeling exacerbated by the penetration of inflammatory cells into the damaged vessel, which in turn creates the activated environment that stimulates the migration of smooth muscle cells (SMC) from the media into the intima. ${ }^{1,2}$ SMC hyperplasia contributes to the formation of the lesion, with loss of lumen diameter and vascular contractility, processes that underpin pathologies such as atherogenesis and restenosis after percutaneous coronary intervention. SMC migration and hyperplasia are preceded by the modulation of SMC phenotype from a contractile state to a synthetic state, characterized by a decrease in the number of myofilaments and contractile proteins and the development of a more epithelioid shape, resembling its embryonic dedifferentiated counterparts. ${ }^{3-5}$ Platelet-derived growth factor subunit B homodimer (PDGF-BB) has been shown to be a potent and selective factor promoting phenotypic switching of SMC into the synthetic state, facilitating its proliferation and migration. ${ }^{6,7}$ Indeed, PDGF-BB expression by macrophages in models of atherogenesis has been shown to mediate SMC migration into the tunica intima. ${ }^{8}$

At the transcriptional level, PDGF-BB effects phenotypic changes by regulating the expression of a number of pathophysiologically relevant genes, one of which is tenascin-C (TN-C; also known as cytotactin), ${ }^{9,10}$ a large extracellular matrix glycoprotein that modulates cell adhesion. TN-C is composed of six polypeptides, each containing epidermal growth factor-like (EGFL) repeats, fibronectin type III (FN III) domains (some conserved and some alternatively spliced), and a C-terminal fibrinogen globe. The N-terminal tenascin assembly domain allows the individual polypeptides to form a hexabrachion structure. ${ }^{11} \mathrm{TN}-\mathrm{C}$ promotes SMC migration either by sterically blocking fibronectin-integrin interactions ${ }^{12}$ or activating components of adhesion complexes, such as PDGFR- $\beta$, $\alpha \vee \beta 3$ integrin, focal adhesion kinase (FAK), and protooncogene tyrosine-protein kinase $\mathrm{Src}$ (SRC). ${ }^{13}$ The

Supported by grants from the National Health and Medical Research Council, Australian Research Council, and the National Heart Foundation. Accepted for publication February 28, 2012.

Address reprint requests to Levon M. Khachigian, Ph.D., Centre for Vascular Research, University of New South Wales, Sydney, NSW 2052, Australia. E-mail: I.khachigian@unsw.edu.au. 
C isoform of tenascin (TN-C) is induced by mechanical injury ${ }^{14}$ and has been found to contribute to intimal hyperplasia in a mouse model of graft artery stenosis. ${ }^{15}$ In addition, TN-C protein is highly expressed in both murine and human atherosclerotic plaques. ${ }^{16,17}$ Several factors have been shown to transcriptionally regulate $\mathrm{TN}-\mathrm{C}$ expression in fibroblasts. ${ }^{18,19}$ Interspecies alignment of the TN-C proximal promoter reveals some conserved transactivation sites that lend themselves to acute up-regulation, including a strain-responsive element, several Ets binding sites, and a homeodomain binding site close to the TATA box. ${ }^{11}$ In human dermal fibroblasts, PDGF-AA activates the TN-C promoter through Sp1, Ets-1, and Ets-2. ${ }^{20}$ However, transcription factors controlling TN-C expression in SMC in the context of vascular injury have not previously been investigated, nor the involvement of the mammalian stress response pathway regulator activating transcription factor 4 (ATF-4) in this critical process. In earlier work, we showed that ATF-4 is induced after SMC injury in vitro and in balloon-injured rat arteries. ${ }^{21,22}$ ATF-4 (also known as CREB-2, TaxREB67, and C/ATF) is a ubiquitous basic/leucine zipper domain (bZIP) transcription factor and a member of the cAMP-responsive element-binding (CREB) protein family. ${ }^{23}$ Here, using knockdown, deficiency, and overexpression strategies, we demonstrate the requirement of ATF-4 in injury- and PDGF-BB-inducible TN-C expression and SMC wound repair.

\section{Materials and Methods}

\section{Cell Culture, Plasmid Construction, and Transient Transfection}

Primary rat aortic smooth muscle cells (RASMCs) were obtained from Cell Applications (San Diego, CA) and cultured in Waymouth's medium, pH 7.4, containing 10\% fetal bovine serum (FBS) and antibiotics as described previously. ${ }^{24}$ Cells were rendered quiescent by incubation in Waymouth's medium without FBS for 24 hours. Cells were not used beyond passage 8 in experiments. Atf4 $^{-/-}$and $\mathrm{AtfH}^{+/+}$mouse embryonic fibroblasts (SV4Otransformed mEFs) were obtained from Dr. David Ron (Skirball Institute of Biomolecular Medicine, New York University School of Medicine) and grown in complete medium consisting of advanced Dulbecco's modified Eagle's medium (DMEM) with nonessential amino acids (InvitrogenLife Technologies, Carlsbad, CA) and 10\% FBS, 10 units $/ \mathrm{mL}$ penicillin, $10 \mu \mathrm{g} / \mathrm{mL}$ streptomycin, 2 mmol/L L-glutamine, and $55 \mu \mathrm{mol} / \mathrm{L} \beta$-mercaptoethanol. The ATF-4pcDNA3 used has been described previously. ${ }^{22}$ For transfection, cells were allowed to grow to $60 \%$ to $70 \%$ confluence, then were transfected with indicated constructs using FUGENE 6 transfection agent (Roche Applied Science-Roche Molecular Biochemicals, Mannheim, Germany) or RNAiMAX transfection reagent (Invitrogen-Life Technologies). For PDGF receptor inhibition, growth-quiescent RASMCs were pretreated with inhibitors to PDGFR- $\beta$ (AG1295, $10 \mu \mathrm{mol} / \mathrm{L} ;$ Calbiochem) or PDGFR- $\alpha$ (ab35765; 1 $\mu \mathrm{g} / \mathrm{mL}$; Abcam, Cambridge, MA) for 1 hour, before growth factor exposure.

\section{Semiquantitative $R T$-PCR}

Total RNA was prepared from cells that were exposed to PDGF-BB (Sigma-Aldrich, St. Louis, MO) with TRIzol reagent in accordance with the manufacturer's instructions (Invitrogen-Life Technologies). RNA was reverse-transcribed to cDNA using oligo(dT) primers and SuperScript II Reverse Transcriptase (Invitrogen-Life Technologies). PCR was performed in a $20 \mu \mathrm{L}$ reaction containing 2.5 $\mathrm{mmol} / \mathrm{L} \mathrm{MgCl} 2,0.1 \mathrm{mmol} / \mathrm{L}$ dNTP, $0.1 \mu \mathrm{mol} / \mathrm{L}$ primers, $1 \mu \mathrm{L}$ cDNA, and $1 \cup$ of Platinum Taq polymerase (Invitrogen-Life Technologies) using a GeneAmp PCR system 2400 thermal cycler (Applied Biosystems-Life Technologies, Foster City, CA). ATF-4 and GAPDH PCR cycling conditions were as described previously. ${ }^{22}$ TN-C primers and cycling conditions used were as described previously. ${ }^{9}$

\section{$R T$-qPCR Assays}

Quantitative real-time RT-PCR (RT-qPCR) was performed using SYBR Green PCR master mix (Applied BiosystemsLife Technologies). Briefly, total RNA was isolated and reverse-transcribed as described above. PCR was performed in a $10 \mu \mathrm{L}$ reaction, consisting of $5 \mu \mathrm{L}$ SYBR Green PCR master mix and $0.3 \mu \mathrm{mol} / \mathrm{L}$ of forward and reverse primers. PCR amplification was performed using a Corbett Rotor-Gene RG-6000 system (Qiagen, Valencia, CA). The PCR conditions consisted of an initial hold step of $50^{\circ} \mathrm{C}$ for 2 minutes and then activation at $95^{\circ} \mathrm{C}$ for 10 minutes, followed by 40 cycles of $95^{\circ} \mathrm{C}$ for $10 \mathrm{sec}-$ onds, $60^{\circ} \mathrm{C}$ for 15 seconds, and $72^{\circ} \mathrm{C}$ for 10 seconds. The $\mathrm{C}_{\mathrm{T}}$ value of ATF-4, Egr-1, JunB, or TN-C amplification was normalized to that of $\beta$-actin control. PCR products were visualized on ethidium bromide-stained agarose gels to ensure product of right size and no primer dimerization. All initial runs were performed with simultaneous generation of efficiency curves. Data were generated from at least triplicate samples. To amplify the different TN-C isoforms, the following RT-GPCR primers were used: for the Large isoform, Tnc_Large_Fwd: 5'-CCAGGAGGACTCAGGTCAGTGGACCTA-3' and Tnc_Large_Rev: 5'-AGG GATCTCCTCTGTCAAGACCTCAAC-3'; for the A1A2 isoform, Tnc_Large_Fwd and A1A2_Rev: 5'-CTTGGGAGAGCCCATGGCTGTCAAGACCTCAAC-3'; for the D-isoform, Tnc_Inter_Fwd: 5'-AAGGCATCCACAGAAGCTGAA-3' and Tnc_Inter_Rev: 5'-GAGGGTTATTTCTTGTGGTTCAGAGTT-3'; for the Small isoform, Tnc_Small_Fwd: 5'-GAAGGCATCCACAGCCATGG-3' and Tnc_Small_Rev: 5'-TCACCAGTCGGGTCTCAGTATC-3'; for Egr-1, Egr-1_Fwd: 5'-GCCTाT TGCCTGTGACATTT-3' and Egr-1 Rev: 5'-AGCCCGGAGAGGAGTAAGAG-3'; for Jun B, JunB Fwd: 5'-CACGACGACTCATACGCAGC-3' and JunB Rev: 5'-GACCCTTGAGACCCCGATAA-3'; and for $\beta$-actin, $\beta$-actin_Fwd: 5'-AGCCATGTACGTAGCCATCC-3' and $\beta$ actin_Rev: 5'-CTCTCAGCTGTGGTGGTGAA-3'. Primers used to amplify ATF-4 were as described previously. ${ }^{22}$

\section{Western Blot Analysis}

RASMCs were cultured in 100-mm tissue culture plates and treated with PDGF-BB for various lengths of time. For collection of cell lysates, cell monolayers were washed 
twice in 1X PBS; total protein was extracted in 150 $\mathrm{mmol} / \mathrm{L} \mathrm{NaCl}, 50 \mathrm{mmol} / \mathrm{L}$ Tris- $\mathrm{HCl}(\mathrm{pH} 7.5), 1 \%$ sodium deoxycholate, $0.1 \%$ SDS, $1 \%$ Triton X-100, $5 \mathrm{mmol} / \mathrm{L}$ EDTA, $10 \mathrm{mg} / \mathrm{mL}$ leupeptin, $1 \%$ aprotinin, and $2 \mathrm{mmol} / \mathrm{L}$ phenylmethylsulfonyl fluoride. For secreted TN-C in the media, $5 \mathrm{~mL}$ of the media was concentrated (Vivaspin concentrator, $30 \mathrm{kDa}$ molecular weight cutoff; GE Healthcare, Little Chalfont, UK) to a final volume of $300 \mu \mathrm{L}$. Protein sample (10 to $20 \mu \mathrm{g}$ of lysate or $50 \mu \mathrm{L}$ concentrated tunica media) was loaded onto a $6 \%$ to $10 \%$ SDSpolyacrylamide gel and electroblotted onto a polyvinylidene difluoride nylon membrane (EMD Millipore, Bedford, MA). Membranes were blocked in 0.05\% Tween $20(\mathrm{v} / \mathrm{v})$ PBS containing 5\% skim milk, then incubated with the following primary antibodies: ATF-4 (sc-22800) and TN-C (sc-20932) from Santa Cruz Biotechnology (SCBT; Santa Cruz, CA) and $\beta$-actin (A5316) from SigmaAldrich. Membranes were then incubated with horseradish peroxidase-conjugated secondary antibodies (Dako, Carpinteria, CA). Proteins were visualized by chemiluminescence detection (NEN Life Sciences, Boston, MA).

\section{Small Interfering RNA and in Vitro Wound Repair Assay}

RASMCs were seeded in Petri dishes and growth was arrested with serum-free medium for 24 hours before transfection with $0.4 \mu \mathrm{mol} / \mathrm{L}$ small interfering RNA (siRNA; Qiagen) targeting endogenous rat ATF-4. ${ }^{22}$ siRNA technology provides an invaluable tool for exploring target gene function. ${ }^{25}$ At 24 hours after serum arrest, ATF-4 or TN-C isoforms were inducibly expressed by the addition of PDGF-BB for the appropriate length of time, and total RNA was extracted and analyzed as described above. For wounding assays, the RASMC or mEF cell monolayer was scratched using a sterile toothpick; the growth medium was changed, and PDGF-BB was added. Wound closure was measured by counting the number of cells in the wound area at 24 hours after the scratch. Samples were prepared in triplicate, and cell counts were taken as the average of three fields per sample under $\times 100$ magnification.

\section{Dual-Chamber Migration Assay}

Atf ${ }^{+/+}$and $\mathrm{Atf}^{-/-} \mathrm{mEFs}$ were grown in minimal-serum medium for 24 hours, before seeding at a density of $5 \times$ $10^{4}$ cells in $0.1 \%$ FBS DMEM in the top chamber of a Millicell cell culture insert (EMD Millipore) with an $8 \mu \mathrm{m}$ pore size for 24-well dual-chamber migration assays. Inserts were coated with TN-C or bovine serum albumin (2 $\mu \mathrm{g} /$ insert). This was achieved by the addition of TN-C or bovine serum albumin in PBS solution to the apical chamber, incubation for 2 hours at $37^{\circ} \mathrm{C}$, and then removal of excess liquid and air-drying in the hood for 30 minutes. Inserts were equilibrated with 0.1\% FBS DMEM before cell seeding. The bottom chamber contained either $0.1 \%$ FBS DMEM or $0.1 \%$ FBS DMEM with $50 \mathrm{ng} / \mathrm{mL}$ PDGF-BB. Three inserts were prepared for each treatment group. The migration assay was allowed to proceed at $37^{\circ} \mathrm{C}$ and
$5 \% \mathrm{CO}_{2}$ for 24 hours, at which point the cells were washed three times with $1 \times$ PBS to dislodge nonmigratory cells and then were fixed in Quick DIP I solution (Thermo Fisher Scientific, Australia) for 15 minutes. The inserts were washed three times with $1 \times$ PBS, stained with Fronine Quick DIP II solution for 15 minutes, and then were washed three more times with $1 \times$ PBS. The inserts were mounted on glass slides using ProLong Gold anti-fade reagent with DAPI (Invitrogen-Life Technologies) and then were visualized under a fluorescent microscope. The number of cells that had migrated through each membrane was determined by counting the number of cells in three randomly selected microscopic fields at $\times 100$ magnification.

\section{Rat Carotid Artery Injury and Immunohistochemistry}

Balloon catheter injury to the carotid artery of adult Sprague Dawley rats (450 to $550 \mathrm{~g}$ ) was performed as described previously. ${ }^{22}$ After injury, $100 \mu \mathrm{L}$ of PBS containing $10 \mu \mathrm{L}$ FUGENE 6 and $50 \mu \mathrm{g}$ siRNA or the vehicle itself was infused into the ligated segment for 20 minutes at $100 \mathrm{~mm} \mathrm{Hg}$. The rats were sacrificed 14 days later. Tissue processing and immunohistochemistry using TN-C antibodies were performed essentially as described previously.22,26 Digital images of sections were captured using a DP-70 Olympus microscope. Relative staining in the neointima was determined using Image version $1.44(\mathrm{NIH}$, Bethesda, MD). The mean integrated density values (measured in pixels) were determined on three representative sections of equal area, using two fields of view from four rats per group.

\section{Replicates and Statistical Analysis}

For data presented in histograms, results are expressed as means \pm SE performed in triplicate or quadruplicate. Data are representative of three to five independent determinations. Data were analyzed for statistical significance $(P<0.05)$ using two-tailed Student's $t$-test or analysis of variance between groups.

\section{Results}

\section{Levels of ATF-4 mRNA and Protein Are Increased in SMCs by Exposure to PDGF-BB}

Building on our previous demonstration that both in vitro cell injury and FGF-2 induce ATF-4 mRNA expression, ${ }^{22}$ we explored the possibility that PDGF-BB, a potent SMC migratory factor, ${ }^{27}$ could regulate ATF-4 expression in SMCs. RT-qPCR analysis of PDGF-BB-treated RASMCs revealed an increase in ATF-4 mRNA levels within 15 minutes after exposure to the growth factor $(50 \mathrm{ng} / \mathrm{mL})$, which peaked at 1 hour (Figure 1A). This induction was dose-responsive, with ATF-4 transcript levels induced at PDGF-BB levels as low as $5 \mathrm{ng} / \mathrm{mL}$ and highest transcript levels induced at 25 to $50 \mathrm{ng} / \mathrm{mL}$ (Figure 1B). Western blot analysis of PDGF-BB-treated RASMCs revealed an increase in ATF-4 protein expression within 30 minutes, peaking at 2 to 4 hours (Figure 1C). Altered expression of 

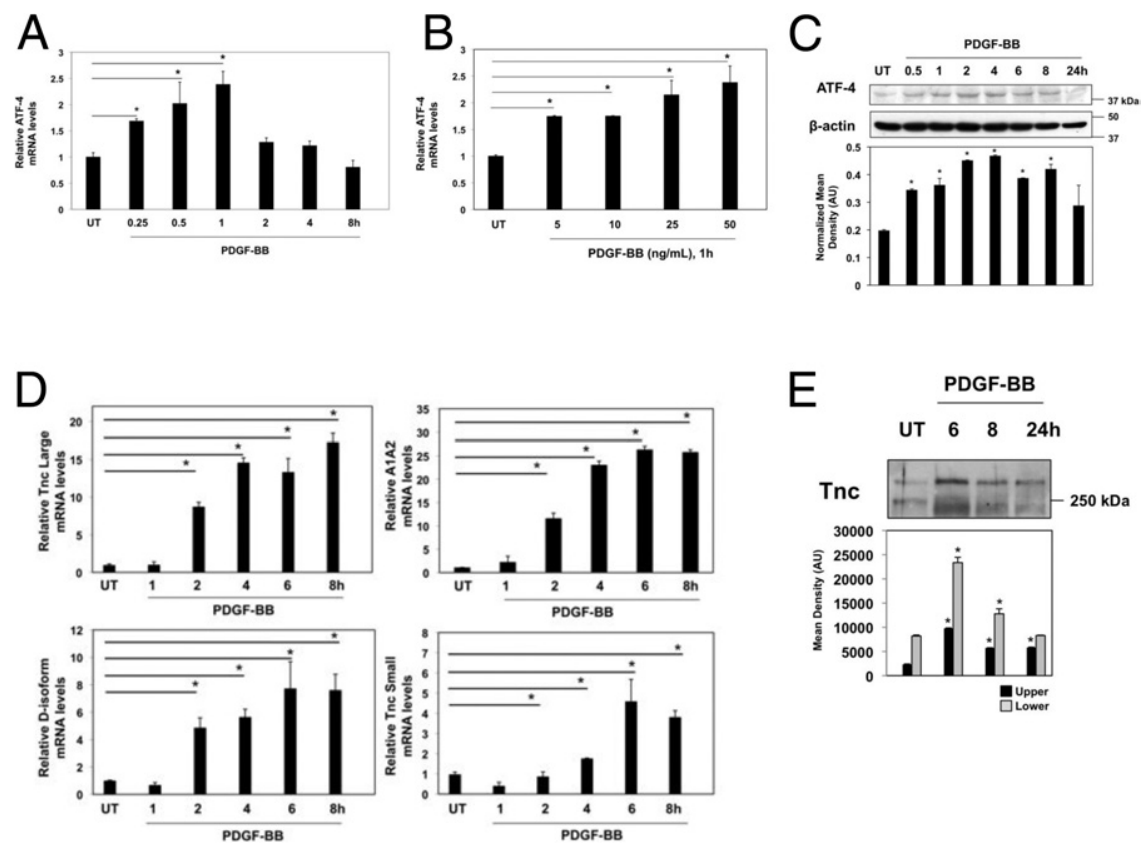

E

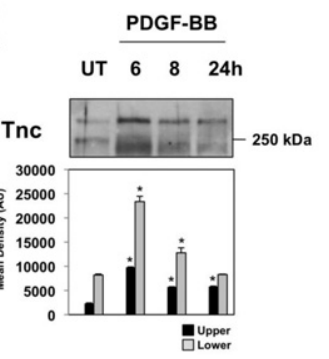

Figure 1. PDGF-BB induces ATF-4 mRNA and protein and TN-C mRNA and secreted protein in growth-quiescent vascular SMCs. A: RASMCs were rendered growth-quiescent by serum deprivation for 24 hours, then exposed to PDGF-BB (50 ng/mL) for various lengths of time. B: RASMCs were treated with various concentrations of PDGF-BB (from 5 to $50 \mathrm{ng} / \mathrm{mL}$ ) for 1 hour, before isolation of total RNA for RT-qPCR analysis of ATF-4. C: Total cell lysates of PDGF-BB (50 ng/mL)-treated RASMCs were used for Western blot analysis with ATF- 4 antibody (sc-22800; SCBT). Western blot for $\beta$-actin (A5316; Sigma-Aldrich) shows unbiased loading. Scanning densitometry, with ATF-4 protein levels normalized to $\beta$-actin. D: RASMCs were treated with PDGF-BB $(50 \mathrm{ng} / \mathrm{mL})$, then harvested at various time points. RT-qPCR analysis was performed using primers to the four TN-C isoforms: Large, A1A2, D-isoform, and Small. Data were normalized to $\beta$-actin. E: Media collected from PDGF-BB-treated RASMCs was concentrated and equal volumes were subjected to Western blot analysis for TN-C (sc-20932; SCBT) Data were analyzed by scanning densitometry. ${ }^{*} P<0.05 . n=3(\mathbf{B}) ; n=4(\mathbf{D})$. Tnc, tenascin-C. this stress response regulator by PDGF-BB has hitherto not been reported in any cell type. PDGF-BB has effects on ATF-4 in non-SMCs. For example, we have observed a profound induction of ATF-4 mRNA levels by PDGF-BB in NIH-3T3 fibroblasts (unpublished data).

\section{PDGF-BB Induces TN-C Expression in SMCs}

From previous microarray analysis comparing differential gene expression in response to injury compromised by the absence of ATF-4, we identified TN-C as a target for investigation. TN-C is expressed in a variety of isoforms through alternative splicing of certain FN III repeats. Primers were designed to detect the four TN-C isoforms expressed in SMC. ${ }^{10} \mathrm{RT}$-qPCR analysis showed that PDGF-BB treatment (50 ng/mL) of RASMCs increased expression of the TN-C Large isoform within 2 hours, reaching nearly 20-fold after 6 to 8 hours (Figure 1D). A similar trend was observed for the three other isoforms: the A1A2, D-isoform, and Small isoforms (Figure 1D), with transcript levels of all four $\mathrm{TN}-\mathrm{C}$ isoforms returning to basal levels by 24 hours (data not shown). Two PDGF-BB inducible TN-C protein variants, 240 and $280 \mathrm{kDa}$, have been shown to contribute to SMC migration. ${ }^{10}$ Consistently, Western blot analysis revealed an increase in the two TN-C protein isoforms in the media by RASMCs treated with PDGF-BB, peaking at 6 hours of growth factor exposure (Figure 1E).

\section{ATF-4 Overexpression Induces TN-C Expression in SMCs}

To investigate the regulatory role of ATF-4 in mediating TN-C transcription, we transfected a CMV-driven expression vector ATF-4-pcDNA3 (20 $\mu \mathrm{g})$ into RASMCs and used its backbone (pcDNA3) as a control. RT-PCR analysis with primers used to characterize TN-C in SMCs ${ }^{9}$ showed that ATF-4 overexpression induced mRNA levels of all four TN-C isoforms detected to date ${ }^{10}$ : Large (1700 bp), A1A2 (1000 bp), D-isoform (650 bp), and Small (360 $\mathrm{bp}$ ), with GAPDH levels showing even loading (Figure 2A). RT-qPCR analysis of the TN-C Large, A1A2, D-isoform, and Small isoforms after 24 hours also revealed a significant induction with ATF-4 overexpression, compared with the backbone alone (Figure 2B). Likewise, Western blot analysis revealed increased TN-C protein levels in the media when ATF-4 was overexpressed (Figure 2C). To our knowledge, this is the first demonstration of ATF-4 regulation of $\mathrm{TN}-\mathrm{C}$ expression.

\section{PDGF-BB Affects ATF-4 and TN-C Induction through the $\beta$-Receptor, Not the $\alpha$-Receptor}

To determine which PDGF receptor mediates PDGFBB-inducible ATF-4 and TN-C expression, we pretreated growth-quiescent RASMCs with inhibitors of PDGFR- $\beta$ (AG1295, $10 \mu \mathrm{mol} / \mathrm{L})$ or PDGFR- $\alpha$ (ab35765, $1 \mu \mathrm{g} / \mathrm{mL}$ ) for 1 hour, before stimulation with PDGF-BB (50 $\mathrm{ng} / \mathrm{mL}$ ) for 1 and 6 hours. RT-qPCR analysis showed that inhibition of PDGFR- $\beta$ significantly inhibited ATF-4 mRNA levels after 1 hour (Figure 3A) and TN-C Large mRNA levels after 6 hours (Figure 3C), whereas PDGFR- $\alpha$ inhibition had no such effect. A similar trend was observed for the other TN-C isoforms (Figure 3C). Efficacy of inhibition of PDGFR- $\beta$ at this concentration of AG1295 was demonstrated by RT-qPCR analysis of a known downstream gene, Egr1 (Figure 3B). Prior studies have also shown incomplete inhibition with the same concentration of AG1295 in the presence of PDGF-BB in the same cell type. ${ }^{28}$ 


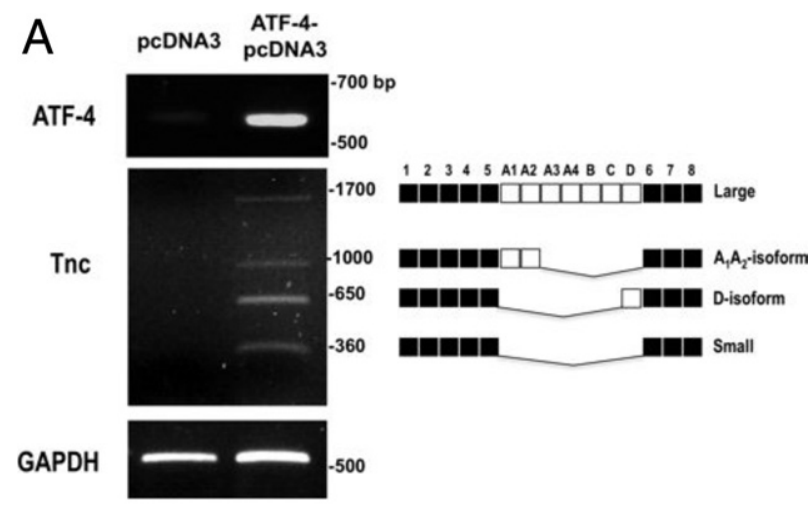

B
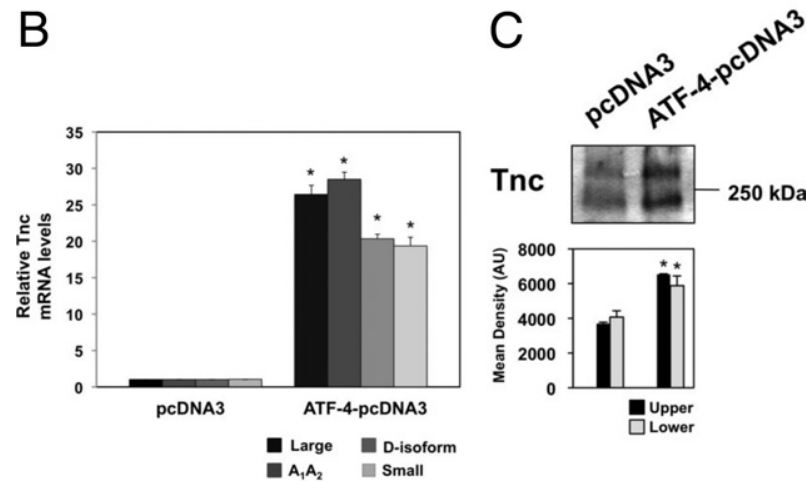

Figure 2. ATF-4 overexpression increases expression of TN-C Large, A1A2, D-isoform, and Small isoforms. A: A cytomegalovirus-driven expression vector ATF-4-pcDNA3 $(20 \mu \mathrm{g})$ was transfected into RASMCs, with the plasmid backbone alone (pcDNA3) used as control. Total RNA was isolated after 24 hours and used for RT-qPCR analysis with primers directed to ATF-4 and TN-C. GAPDH levels show unbiased loading. TN-C isoforms are detailed at the right (diagram adapted from Wallner et $\mathrm{al}^{10}$ ). B: RT-qPCR analysis using primers targeting the four TN-C isoforms. Data were normalized to $\beta$-actin. $\mathbf{C}$ : Western blot analysis for TN-C (sc-20932; SCBT) in media collected from RASMCs transfected with pcDNA3 or ATF-4-pcDNA3. Data were analyzed by scanning densitometry. ${ }^{*} P<0.05 . n=3(\mathbf{A}) ; n=4$ (B).

\section{ATF-4 siRNA Blocks PDGF-BB-Inducible ATF-4 and TN-C mRNA Levels in SMCs}

To examine the effect of silencing ATF-4 on PDGF-BBinducible TN-C expression, we transfected RASMCs with siRNA targeting endogenous rat ATF-4 (0.4 $\mu \mathrm{mol} / \mathrm{L}$ siRNA). The scrambled counterpart (ATF-4 scr) served as a control. The siRNA blocked PDGF-BB (50 ng/mL) induction of ATF-4 after 1 hour, whereas ATF-4 scr had no inhibitory effect (Figure 4A). Importantly, ATF-4 siRNA blocked PDGF-BB-inducible expression of all four TN-C mRNA isoforms after 8 hours, with no concomitant effect of ATF-4 scr (Figure 4B). Selectivity of the ATF-4 knockdown was demonstrated by lack of inhibition of PDGFBB-inducible JunB mRNA levels with ATF-4 siRNA pretreatment (Figure 4 C).

\section{ATF-4 Deficiency Blocks PDGF-BB-Inducible TN-C Transcription}

To provide confirmatory evidence of a pivotal role for ATF-4 in PDGF-BB-inducible TN-C transcription, we performed experiments in an inhibitor-free system, using SV40-transformed mEFs derived from $\mathrm{Atf}^{+/+}$and
Atf4 ${ }^{-1-}$ mice. RT-qPCR analysis revealed a significant reduction in PDGF-BB-inducible TN-C Large transcript levels in Atf4 $^{-/-} \mathrm{mEFs}$, compared with $\mathrm{Atf}^{+/+} \mathrm{mEFs}$, at all time points between 1 and 6 hours after exposure to the growth factor (Figure 4D). PDGF-BB-inducible expression of A1A2, D-isoform, and Small TN-C isoforms was also reduced in the ATF-4-deficient mEFs (Figure 4D).

\section{ATF-4 siRNA Inhibits TN-C Expression in Balloon-Injured Carotid Arteries}

Our previous findings from the rat carotid balloon injury model indicate that intraluminal infusion of an siRNA targeting endogenous ATF-4 blocks injury-inducible ATF-4 protein expression at 4 hours after injury and blocks intimal hyperplasia at 14 days. ${ }^{22}$ To demonstrate a regulatory role for ATF-4 in TN-C expression in vivo, we performed immunohistochemical analysis on the same set of balloon-injured left common carotid rat arteries infused intraluminally with ATF-4 siRNA $(50 \mu \mathrm{g})$, which resulted in ATF-4 knockdown and reduced neointima formation. ${ }^{22}$ TN-C protein expression is weak in uninjured arteries, but is induced within 3 to 4 days, and 1 to 2 weeks after balloon injury. ${ }^{10}$ Although there was less neointima formation in the arteries that were treated with ATF-4 siRNA, densitometric analysis in the neointima 14 days after balloon injury showed that ATF-4 siRNA reduced TN-C expression, compared with the scrambled counterpart (Figure 4E).

\section{ATF-4 Knockdown or Deficiency Blocks PDGF- BB-Inducible SMC and mEF Wound Repair}

We evaluated the biological effect of ATF-4 knockdown in both cell types using an in vitro wound closure assay and dual-chamber migration assay. ATF-4 siRNA (0.4 $\mu \mathrm{mol} / \mathrm{L}$ siRNA) inhibited the PDGF-BB-inducible reparative response after 24 hours (Figure 5, A and C). In contrast, ATF-4 scr had no effect (Figure 5, A and C). When we compared $\mathrm{Atf4}^{+/+}$and $\mathrm{Atf}^{-/-} \mathrm{mEFs}$, we found that the absence of ATF-4 perturbed PDGF-BB-inducible repopulation into the wound area after 24 hours (Figure 5, B and D). We found a similar effect with pretreatment of both Atf4 $^{+/+}$and Atf4 $^{-/-} \mathrm{mEFs}$ with mitomycin $\mathrm{C}^{29}(10 \mu \mathrm{mol} / \mathrm{L}$, data not shown), suggesting that ATF-4 plays a migratory rather than proliferative role in this setting. To further confirm the contribution of ATF-4 to cell migration, we used a dual-chamber model. Migration of mEFs in response to PDGF-BB (50 ng/mL) was significantly reduced in the $\mathrm{Atf}^{4^{-1}-} \mathrm{mEFs}$, compared with their wild-type counterparts (Figure 5E). This effect was rescued when the inserts were coated with TN-C (Figure 5E). Thus, these findings from ATF-4 knockdown, deficiency, and overexpression strategies collectively demonstrate the requirement of ATF-4 in injury- and PDGF-BB-inducible $\mathrm{TN}-\mathrm{C}$ expression and SMC and mEF migration.

\section{Discussion}

The present study demonstrates for the first time that the stress response pathway regulator ${ }^{30}$ ATF-4 is dose-de- 


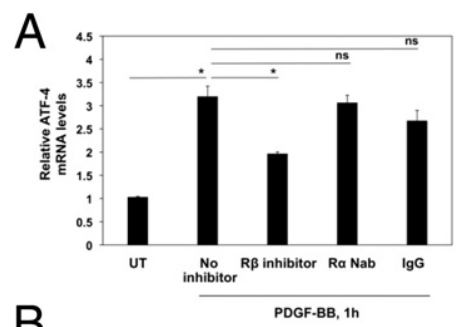

B

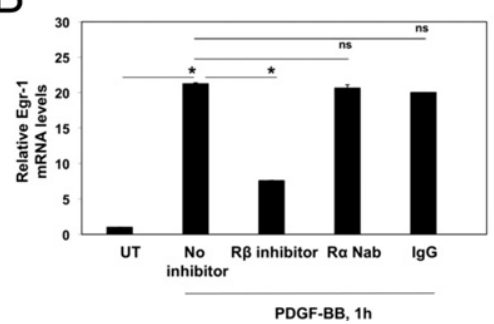

C
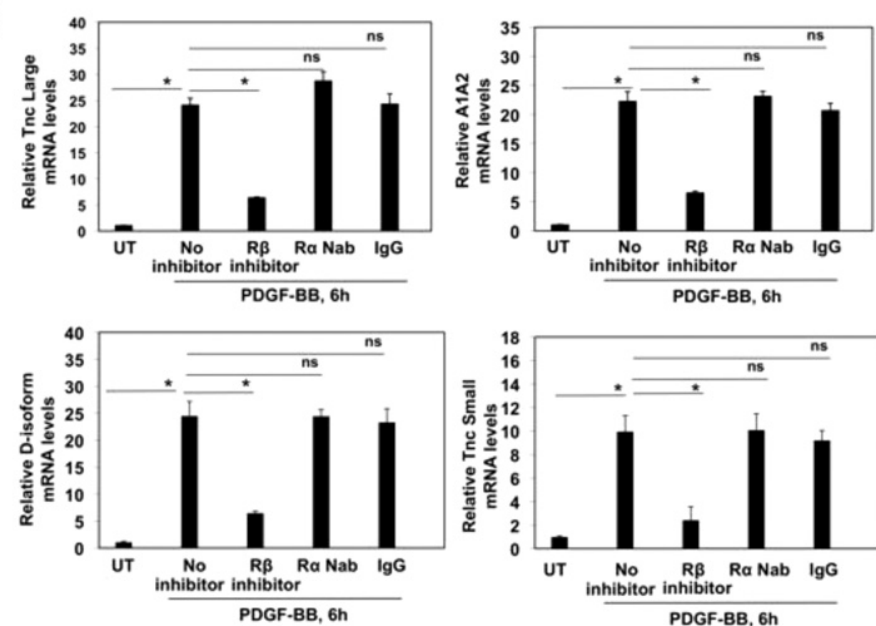

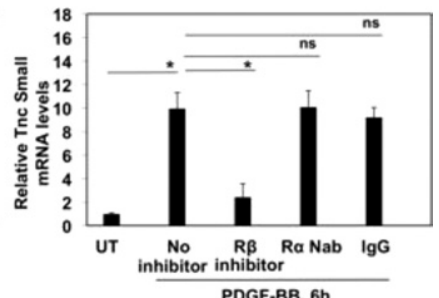

PDGF-BB, 6h

Figure 3. PDGF $\beta$-receptor is required for PDGF-BB up-regulation of ATF- 4 and TN-C. A: RASMCs were rendered growth quiescent for 24 hours, then were treated with a small-molecule inhibitor to the $\beta$-receptor (AG1295, $10 \mu \mathrm{mol} / \mathrm{L}$; Calbiochem), and a neutralizing antibody (Nab) to the $\alpha$-receptor (ab35765; $1 \mu \mathrm{g} / \mathrm{mL}$; Abcam) for 1 hour. IgG served as a control. RASMCs were then treated with PDGF-BB (50 ng/mL) for 1 hour, total RNA was collected, and RT-qPCR analysis performed using primers directed to ATF- 4 . Data were normalized to $\beta$-actin. Expression of PDGFR- $\alpha$ and PDGFR- $\beta$ in these cells was confirmed by Western blotting (data not shown). B: RT-qPCR analysis for the Egr-1 gene, which lies downstream of the PDGFR- $\beta$ gene. C: RASMCs were pretreated with inhibitors for 1 hour and then with PDGF-BB for 6 hours. Total RNA was collected, and TN-C mRNA levels were determined by RT-qPCR analysis. Data were normalized to $\beta$-actin. * $P<0.05$. $n=4$.

pendently induced by PDGF-BB in vascular SMCs through PDGFR- $\beta$, and that ATF-4 positively regulates the expression of the extracellular matrix glycoprotein TN-C. We further show that absence of ATF-4 disables PDGF-BB-inducible TN-C expression and wound repair mechanisms in vitro, in both vascular SMCs and mEFs. Finally, we provide evidence that ATF-4 silencing abrogates TN-C expression in SMCs of rat carotid arteries 14 days after balloon injury.

The transient induction of ATF-4 by PDGF-BB in RASMCs and fibroblasts is characteristic of immediate-

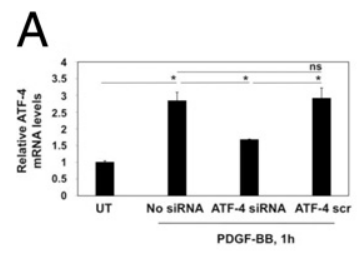

B
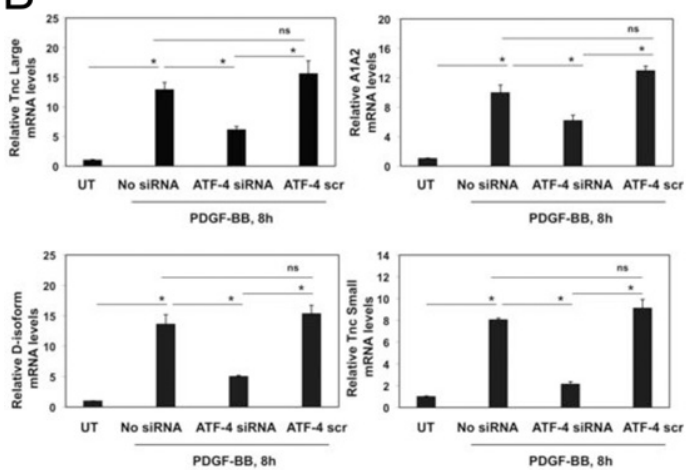

D
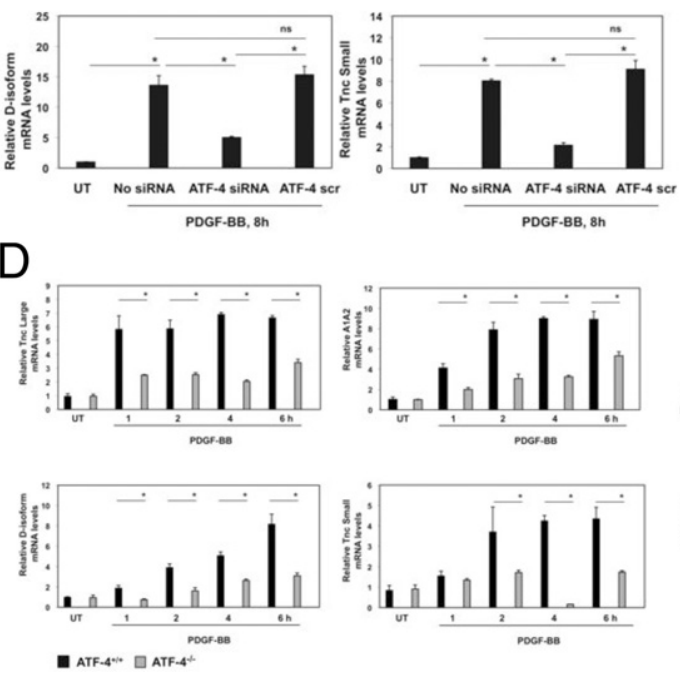

E
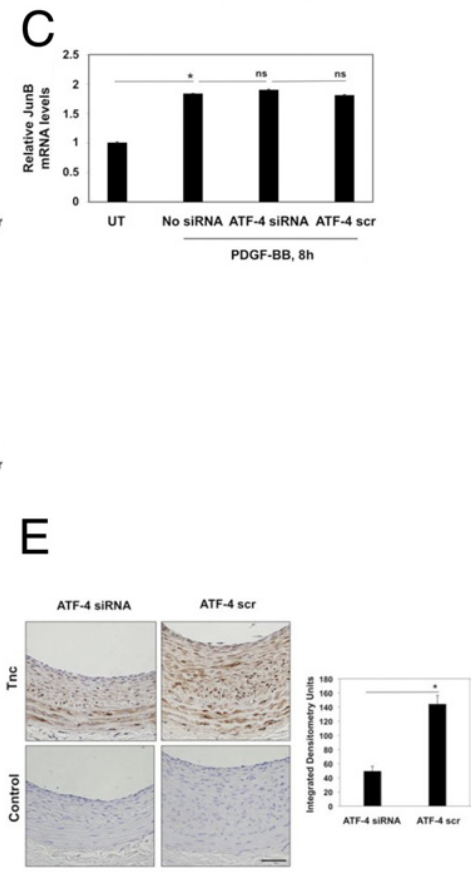

Figure 4. ATF-4 is required for PDGF-BB-inducible TN-C transcript levels in vitro and in jury-inducible protein expression in vivo. A: RASMCs were transfected with $0.4 \mu \mathrm{mol} / \mathrm{L}$ siRNA targeting endogenous ATF-4 (Qiagen), before exposure to PDGF-BB $(50 \mathrm{ng} / \mathrm{mL})$ for 1 hour. The scrambled counterpart (ATF-4 scr) used at the same concentration served as a negative control. Total RNA was extracted and used for RT-qPCR analysis. Data were normalized to $\beta$-actin. $\mathbf{B}$ and $\mathbf{C}$ : RASMCs were transfected with ATF-4 siRNA or ATF-4 scr before PDGF-BB treatment $(50 \mathrm{ng} / \mathrm{mL})$ for 8 hours. Total RNA was extracted and used for RTqPCR analysis of the four TN-C isoforms (B) or JunB (C). D: SV40-transformed mEFs $\left(\mathrm{Atf}^{+/ /+}\right.$ and $\left.\mathrm{Atf}^{-/}\right)$were treated with PDGF-BB (50 $\mathrm{ng} / \mathrm{mL}$ ); total RNA was isolated at different times, then used for RT-qPCR analysis for the four TN-C isoforms. Data were normalized to $\beta$-actin. E: Rat carotid arteries were ballooninjured, then perfused with ATF-4 siRNA (50 $\mu \mathrm{g})$ or the scrambled control. After 14 days, arteries were harvested and sections $(5 \mu \mathrm{m})$ were stained for TN-C immunoreactivity using TN-C antibodies (sc-20932; SCBT). Representative sections are shown at left; quantification of TN-C staining in the neointima is shown at right. Control images show lack of specific signal when the primary antibody is omitted. Scale bar $=50 \mu \mathrm{m} .{ }^{*} P<0.05 . n=4$. 
A

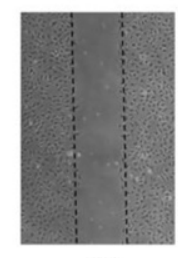

UT

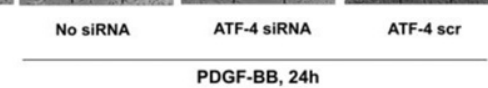

B

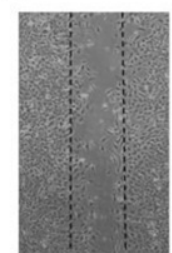

UT

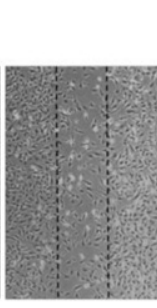

PDGF-BB, 24h
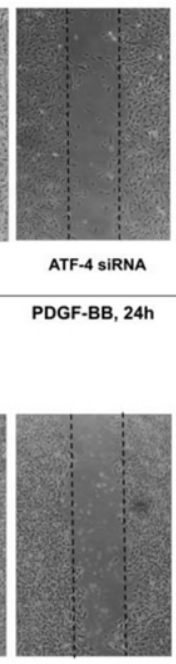

UT
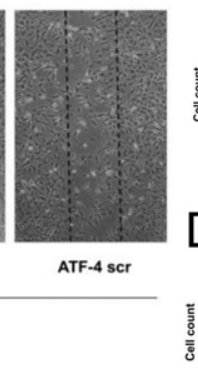

ATF-4+ mEFs

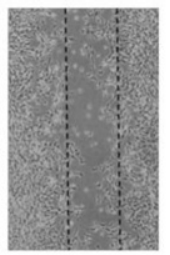

PDGF-BB EFs

ATF $-4^{+* *} \mathrm{mEFs}$

PDGF-BB

s

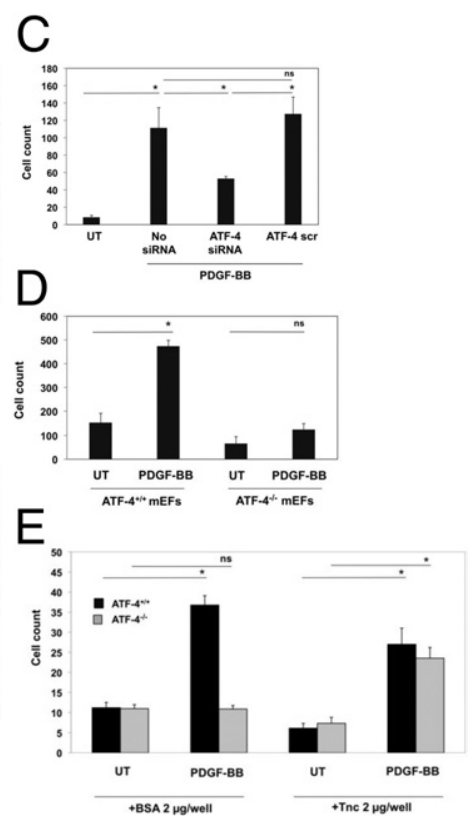

$\mathrm{E}$

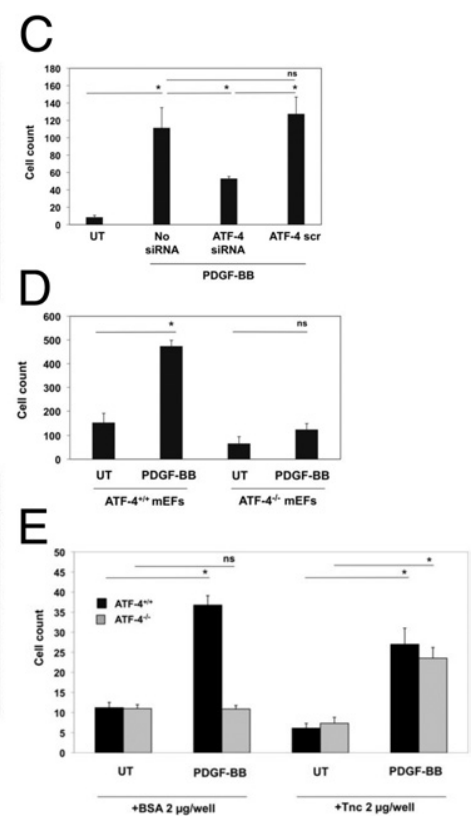

Figure 5. ATF- 4 knockdown or deficiency inhibits PDGF-BB-inducible wound repair. A Growth-quiescent SMCs were transfected with $0.4 \mu \mathrm{mol} / \mathrm{L}$ ATF- 4 siRNA or ATF- 4 scr. At 24 hours after transfection, cell monolayers were injured using a sterile toothpick, and the cells were incubated with PDGF-BB $(50 \mathrm{ng} / \mathrm{mL})$. B Atf4 $4^{+/+}$and $A t f 4^{-/-}$mEFs were used in the wound closure assay. C: Cell numbers in the denuded zone were quantified 24 hours after scratch injury. Samples were prepared in triplicate, and counts represent the average of three fields per sample. D: The cell population in the denuded zone was quantified 24 hours after incubation with PDGF-BB $(50 \mathrm{ng} / \mathrm{mL})$. E: Growthquiescent mEFs were seeded at equal density into the top chamber of an $8-\mu \mathrm{m}$ pore cell culture insert, coated with either TN-C or bovine serum albumin $(2 \mu \mathrm{g} /$ well $)$, and then placed in 24-well plates containing minimal-serum medium or PDGF-BB $(50 \mathrm{ng} / \mathrm{mL})$. Cell migration was measured after 24 hours. Migration was quantified by counting the number of cells in five randomly selected microscopic fields. Original magnification, $\times 100 .{ }^{*} P<0.05$; ns, not significant. early genes, whose acute expression triggers the activation of other genes that effect critical changes in the vessel wall, through autocrine mechanisms of growth and proliferation. ${ }^{31}$ The novelty of the present work lies in the link established here between ATF-4 and TN-C, a known regulator of cellular adhesion and motility. TN-C plays a pivotal role in processes requiring cell movement, such as normal fetal development, and is minimally expressed in most adult tissues. Its expression is also up-regulated in conditions such as inflammation, wound healing, and cancer. In particular, it has been implicated in tumor cell proliferation, metastasis, and angiogenesis. ${ }^{32}$ In the context of SMCs, the TNC gene was identified as one of three genes significantly induced by mechanical $\operatorname{strain}^{14}$ and was found to be inducible by PDGF-BB. ${ }^{9,10}$ Initial studies using cultured SMCs that were exposed to PDGF-BB revealed three $\mathrm{TN}-\mathrm{C}$ transcript isoforms by $\mathrm{RNase}$ protection assay: Large, D-isoform (intermediate), and Small isoforms. Functional studies demonstrate that the unspliced form of human TN-C inhibits adhesion of human and RASMCs to fibronectin in a concentration-dependent manner. ${ }^{9}$ Later, another TN-C isoform was detected, containing the $\mathrm{A} 1$ and $\mathrm{A} 2$ repeats of the $\mathrm{FN}-\mathrm{III}$ domain. ${ }^{10}$ This A1A2 protein was observed to be the predominant TN-C isoform expressed in rat pup SMCs and neointimal SMCs. Furthermore, among the four isoforms expressed in cultured SMCs, chemotactic activity has been attributed only to the $\mathrm{A} 1 \mathrm{~A} 2$ protein, which can trigger SMC migration levels, similar to PDGF-BB. ${ }^{10}$ Findings presented here confirm that PDGF-BB stimulates mRNA expression of all four TN-C isoforms in cultured RASMCs and that ATF-4 overexpression in these cells can induce mRNA levels of all four TN-C isoforms, containing various repeats of the A-D FN III domain. It is the presence of this alternatively spliced A-D FN III domain that imparts to the TN-C molecule its counteradhesive and migratory properties. ${ }^{9,10}$ In contrast, the absence of these FN III repeats in smaller TN-C variants is associated with the production of a stable extracellular matrix in more quiescent tissues. ${ }^{11}$

TN-C mRNA levels are increased in rat carotid arteries 2 weeks after balloon injury, with expression most significant in neointimal SMCs. ${ }^{12}$ Functionally, TN-C contributes to intimal hyperplasia after aortotomy, with TN-C-deficient mice exhibiting significant reductions in neointima/media ratios, proliferating cell nuclear antigen (PCNA) index values, and Alcian Blue-positive proteoglycans. ${ }^{33}$ This is important, because we have previously shown that ATF-4 is required for neointima formation in balloon-injured rat carotid arteries. $^{22}$ The present study shows that ATF-4 knockdown or deficiency blocks PDGF-BB induction of TN-C in SMCs and mEFs. Importantly, it shows that blockade of ATF-4 inhibits TN-C protein expression in the neointima 14 days after balloon injury. In addition, it demonstrates the requirement of ATF-4 for migration in response to PDGFBB. Moreover, PDGF-BB-induced migration was also compromised in ATF-4 null mEFs, which was rescued by addition of TN-C. This finding is in line with recent findings showing that TN-C augments PDGF-BB stimulation of SMC migration by enhancing crosstalk signaling between PDGFR- $\beta$ and $\alpha \vee \beta 3$ integrin through increased SRC recruitment and FAK activation, which suggests that TN-C is part of an autocrine mechanism whereby growth factors involved in the response of SMC to injury can further modulate cell behavior. ${ }^{13}$ Our present findings suggest that ATF-4 regulation could be upstream to this process.

Together, the data presented here demonstrate PDGF-BB control of ATF-4 through the $\beta$-receptor and ATF-4 control of $\mathrm{TN}-\mathrm{C}$ expression in vitro and in vivo, and also demonstrate the requirement of ATF-4 for cell migration in response to PDGF-BB.

Regulation by ATF-4 of neointima formation after injury is likely to extend beyond this axis. Indeed, our previous demonstration of FGF-2-dependent ATF-4 induction and the ATF-4 siRNA blockade of intimal thickening ${ }^{22}$ sup- 
ports this possibility and, taken together with our present demonstration that ATF-4 mediates TN-C expression and wound repair inducible by injury and PDGF-BB, suggests that ATF-4 may represent an important target for future interventional therapy in a range of vasculo-occlusive pathologies involving SMCs. Given that TN-C has been implicated in cancer progression and metastasis, ATF-4 is implicated in these processes as well.

\section{Acknowledgments}

We thank Prof. Alex Bobik and Peter Kanellakis (Baker IDI Institute, Melbourne, Australia) for providing injured rat tissue, Dr. David Ron (Skirball Institute of Biomolecular Medicine, New York University Langone Medical Center) for kindly providing $\mathrm{Atf}^{+/+}$and $\mathrm{Atf}^{-/-} \mathrm{mEFs}$, Gavin McKenzie (University of New South Wales Histology and Microscopy Unit) for technical assistance, Dr. Lionel Lourenco-Dias and Annie Au-Yeung (Centre for Vascular Research, University of New South Wales) for helpful comments, and Dr. Lucinda McRobb (Centre for Vascular Research, University of New South Wales) for assistance with data analysis.

\section{References}

1. Libby P, Ridker PM, Maseri A: Inflammation and atherosclerosis. Circulation 2002, 105:1135-1143

2. Libby P, Schwartz SM, Brogi E, Tanaka H, Clinton S: A cascade model for restenosis. Circ Res 1992, 86(6 Suppl):III47-III52

3. Willis Al, Pierre-Paul D, Sumpio BE, Gahtan V: Vascular smooth muscle cell migration: current research and clinical implications. Vasc Endovascular Surg 2004, 38:11-23

4. Campbell GR, Campbell JH: Smooth muscle phenotypic changes in arterial wall homeostasis: implications for the pathogenesis of atherosclerosis. Exp Mol Pathol 1985, 42:139-162

5. Campbell JH, Campbell GR: The role of smooth muscle cells in atherosclerosis. Curr Opin Lipidol 1994, 5:323-330

6. Owens GK, Kumar MS, Wamhoff BR: Molecular regulation of vascular smooth muscle cell differentiation in development and disease. Physiol Rev 2004, 84:767-801

7. Yoshida T, Gan Q, Shang Y, Owens GK: Platelet-derived growth factor-BB represses smooth muscle cell marker genes via changes in binding of MKL factors and histone deacetylases to their promoters. Am J Physiol Cell Physiol 2007, 292:C886-C895

8. Newby AC, Zaltsman AB: Fibrous cap formation or destruction-the critical importance of vascular smooth muscle cell proliferation, migration and matrix formation. Cardiovasc Res 1999, 41:345-360

9. LaFleur DW, Fagin JA, Forrester JS, Rubin SA, Sharifi BG: Cloning and characterization of alternatively spliced isoforms of rat tenascin. Platelet-derived growth factor-BB markedly stimulates expression of spliced variants of tenascin mRNA in arterial smooth muscle cells. J Biol Chem 1994, 269:20757-20763

10. Wallner K, Shah PK, Sharifi BG: Balloon catheterization induces arterial expression of new tenascin-C isoform. Atherosclerosis 2002, 161:75-83

11. Jones PL, Jones FS: Tenascin-C in development and disease: gene regulation and cell function. Matrix Biol 2000, 19:581-596

12. Majesky MW: Neointima formation after acute vascular injury. Role of counteradhesive extracellular matrix proteins. Tex Heart Inst J 1994, $21: 78-85$

13. Ishigaki T, Imanaka-Yoshida K, Shimojo N, Matsushima S, Taki W, Yoshida T: Tenascin-C enhances crosstalk signaling of integrin alphavbeta3/PDGFR-beta complex by SRC recruitment promoting PDGF-induced proliferation and migration in smooth muscle cells. J Cell Physiol 2011, 2216:2617-2624

14. Feng $\mathrm{Y}$, Yang JH, Huang H, Kennedy SP, Turi TG, Thompson JF, Libby $P$, Lee RT: Transcriptional profile of mechanically induced genes in human vascular smooth muscle cells. Circ Res 1999, 85:1118-1123
15. Sawada Y, Onoda K, Imanaka-Yoshida K, Maruyama J, Yamamoto K, Yoshida T, Shimpo H: Tenascin-C synthesized in both donor grafts and recipients accelerates artery graft stenosis. Cardiovasc Res 2007, 74:366-376

16. von Lukowicz T, Silacci M, Wyss MT, Trachsel E, Lohmann C, Buck A, Luscher TF, Neri D, Matter CM: Human antibody against $C$ domain of tenascin-C visualizes murine atherosclerotic plaques ex vivo. J Nucl Med 2007, 48:582-587

17. Pedretti M, Rancic Z, Soltermann A, Herzog BA, Schliemann C, Lachat M, Neri D, Kaufmann PA: Comparative immunohistochemical staining of atherosclerotic plaques using F16, F8 and L19: three clinical-grade fully human antibodies. Atherosclerosis 2010, 208:382-389

18. McKean DM, Sisbarro L, llic D, Kaplan-Alburquerque N, Nemenoff R, Weiser-Evans M, Kern MJ, Jones PL: FAK induces expression of Prx1 to promote tenascin-C-dependent fibroblast migration. J Cell Biol 2003, 161:393-402

19. Jinnin M, Ihn H, Asano Y, Yamane K, Trojanowska M, Tamaki K: Tenascin-C upregulation by transforming growth factor-beta in human dermal fibroblasts involves Smad3, Sp1, and Ets1. Oncogene 2004, 23:1656-1667

20. Jinnin M, Ihn H, Asano Y, Yamane K, Trojanowska M, Tamaki K: Platelet derived growth factor induced tenascin- $C$ transcription is phosphoinositide 3-kinase/Akt-dependent and mediated by Ets family transcription factors. J Cell Physiol 2006, 206:718-727

21. Malabanan KP, Khachigian LM: Activating transcription factor-4 and the acute vascular response to injury. J Mol Med 2010, 88:545-552

22. Malabanan K, Kanellakis P, Bobik A, Khachigian LM: ATF-4 induced by FGF-2 regulates VEGF-A transcription in vascular SMCs and mediates intimal thickening in rat arteries following balloon injury. Circ Res 2008, 103:378-387

23. Lee KA, Hai TY, SivaRaman L, Thimmappaya B, Hurst HC, Jones NC, Green MR: A cellular protein, activating transcription factor, activates transcription of multiple E1A-inducible adenovirus early promoters. Proc Natl Acad Sci USA 1987, 84:8355-8359

24. Khachigian LM, Owensby DA, Chesterman CN: A tyrosinated peptide representing the alternatively spliced exon of the platelet-derived growth factor A-chain binds specifically to cultured cells and interferes with binding of several growth factors [Erratum appeared in J Biol Chem 1992, 267:6449] J Biol Chem 1992, 267:1660-1666

25. Bhindi R, Fahmy RG, Lowe HC, Chesterman CN, Dass CR, Cairns MJ, Saravolac EG, Sun LQ, Khachigian LM: Brothers in arms: DNA enzymes, short interfering RNA, and the emerging wave of small-molecule nucleic acid-based gene-silencing strategies. Am J Pathol 2007 , 171:1079-1088

26. Santiago FS, Ishii H, Shafi S, Khurana R, Kanellakis $P$, Bhindi $R$, Ramirez M, Bobik A, Martin J, Chesterman CN, Zachary I, Khachigian L: Yin Yang- 1 inhibits vascular smooth muscle cell growth and intimal thickening by repressing p21WAF1/Cip 1 transcription and p21WAF1/ Cip1-Cdk4-cyclin D1 assembly. Circ Res 2007, 101:146-155

27. Jawien A, Bowen-Pope DF, Lindner V, Schwartz SM, Clowes AW: Platelet-derived growth factor promotes smooth muscle migration and intimal thickening in a rat model of balloon angioplasty. J Clin Invest 1992, 89:507-511

28. Mochizuki S, Brassart B, Hinek A: Signaling pathways transduced through the elastin receptor facilitate proliferation of arterial smooth muscle cells. J Biol Chem 2002, 277:44854-44863

29. Dewor M, Steffens G, Krohn R, Weber C, Baron J, Bernhagen J: Macrophage migration inhibitory factor (MIF) promotes fibroblast migration in scratch-wounded monolayers in vitro. FEBS Lett 2007. 581:4734-4742

30. Rutkowski DT, Kaufman RJ: All roads lead to ATF4. Dev Cell 2003 4:442-444

31. Miano JM, Vlasic N, Tota RR, Stemerman MB: Smooth muscle cell immediate-early gene and growth factor activation follows vascular injury. A putative in vivo mechanism for autocrine growth. Arterioscler Thromb 1993, 13:211-219

32. Orend G, Chiquet-Ehrismann R: Tenascin-C induced signaling in cancer. Cancer Lett 2006, 244:143-163

33. Yamamoto K, Onoda K, Sawada Y, Fujinaga K, Imanaka-Yoshida K, Shimpo H. Yoshida T, Yada I: Tenascin-C is an essential factor for neointimal hyperplasia after aortotomy in mice. Cardiovasc Res 2005, 65:737-742 